\title{
Teleconsultas Mediadas por Vídeo em Tempos de COVID-19: Uma Revisão Sistemática da Literatura
}

\author{
Rhaylson Silva do Nascimento ${ }^{1}$, \\ Leiva Casemiro de Oliveira ${ }^{1}$, Daniel Faustino Lacerda de Souza ${ }^{1}$ \\ ${ }^{1}$ Programa de Pós-Graduação em Ciência da Computação \\ (UFERSA-UERN) Mossoró - RN - Brazil \\ rhaylson.nascimentodalunos.ufersa.edu.br, \\ \{leiva.casemiro, danielfaustino\}eufersa.edu.br
}

\begin{abstract}
This paper presents a systematic review on the use of information technologies in the mediation of medical consultations by video during the pandemic caused by the new coronavirus. The review aims to identify some of the platforms that have been used in this type of care in Brazil and worldwide, the advantages and limitations of this practice, as well as legal aspects related to the protection of patient data and consultation. Studies available on the CAPES, IEEE and Web of Science repositories published in 2020 were reviewed.
\end{abstract}

Resumo. Este artigo apresenta uma revisão sistemática sobre o uso de tecnologias da informação na mediação de consultas médicas por vídeo durante a pandemia causada pelo novo coronavírus. A revisão tem como objetivo identificar algumas das plataformas que vêm sendo empregadas nesse tipo de atendimento no Brasil e no mundo, os benefícios e limitações dessa prática, bem como aspectos legais relacionados à proteção de dados do paciente e da consulta. Foram revisados estudos disponíveis no portal de periódicos CAPES, IEEE e Web of Science publicados no ano de 2020.

\section{Introdução}

O surgimento do novo coronavírus (SARS-CoV-2), capaz de causar pneumonias graves, fez com que toda a sociedade fosse obrigada a adotar medidas de isolamento, visando a contenção do contágio da doença. Por conta disso, muitas das atividades cotidianas foram impactadas e necessitaram passar por uma readequação, migrando para o ambiente digital. Durante todo o ano de 2020, hospitais do Brasil e do mundo sofreram as consequências de uma demanda crescente de pacientes infectados pelo novo coronavírus, estando a maior parte dos seus recursos humanos e financeiros direcionados para o combate da doença. Para evitar o contágio desnecessário de pessoas que necessitam de atenção básica à saúde, é recomendado que sejam adotadas alternativas de atendimento não presenciais, mediadas por tecnologia da informação e comunicação (TIC) [Medeiros et al. 2020]. Tal prática, denominada telemedicina, provê atendimento especializado em saúde utilizando equipamentos digitais, softwares e agentes humanos de forma remota [Feitosa 2019].

No Brasil, a telemedicina apesar de não ser uma prática nova, se limitava à comunicação médico-médico, à análise de exames a distância e realização de cirurgias remotas. $\mathrm{O}$ atendimento médico, na forma de teleconsultas, não era regulamentado. No 
ano de 2018, houve uma tentativa de regular essa prática, por meio da Resolução CFM 2.227/2018, mas que acabou sendo revogada no ano seguinte por pressão de entidades médicas [Feitosa 2019]. Com a decretação do estado de emergência, em fevereiro de 2020, os conselhos Federais de Medicina e Farmácia, em conjunto com o congresso nacional, elaboraram, em caráter emergencial, normativas que regulamentam o atendimento médico de forma não presencial no país. Como resultado desse debate, foi promulgada a Portaria $\mathrm{n}^{\circ} 467$, de 20 de março de 2020 e a Lei ${ }^{\circ}$ 13.989, de 15 de abril de 2020, ficando estabelecido que as práticas de teleconsulta, telemonitoramento, teleorientação e troca de informações entre médicos e pacientes poderiam se dar de forma totalmente remota.

Com o objetivo de identificar aspectos humanos, tecnológicos e legais envolvidos na realização de teleconsultas mediadas por vídeo e a fim de detectar novas contribuições para o desenvolvimento de softwares para gestão desse tipo de processo, realizou-se uma revisão sistemática de literatura. Foram utilizados três repositórios (CAPES, IEEE e Web of Science) para o levantamento de trabalhos sobre o tema, bem como critérios de busca, inclusão, exclusão e análise da qualidade dos textos. De um conjunto de 108 trabalhos levantados inicialmente, publicados entre os anos de 2018 e 2020, restaram 10 que atenderam a todos os critérios da revisão sistemática.

\section{Protocolo de Revisão Sistemática da Literatura}

Segundo Dresch et al. (2015), a revisão sistemática de literatura (RSL) é uma grande aliada do pesquisador, pois permite a síntese dos diversos estudos sobre determinada temática, através da agregação do conhecimento já publicado. Como resultado desse processo, obtêm-se uma visão mais abrangente do tema, tornando possível a identificação de possíveis lacunas, geradoras de novas pesquisas. O processo de RSL define um conjunto de atividades a serem seguidas para se chegar ao resultado final, executadas de maneira sequencial [Dresch et al. 2015].

\subsection{Questões da Pesquisa}

O objetivo dessa revisão é identificar quais as recomendações para a disponibilização de teleconsultas médicas por videochamadas, suas limitações, bem como critérios relacionados à legislação e tipos de plataformas que vêm sendo utilizadas por médicos e pacientes nesse tipo de atendimento. No Brasil, a teleconsulta só passou a ser permitida a partir de março de 2020, assim, é um tema novo e que merece ser analisado. Para atingir os objetivos dessa revisão, buscou-se responder aos seguintes questionamento de pesquisa: Qual o impacto e as limitações do uso de atendimento médico realizado por meio de videochamadas? A que tipo de regulações esse tipo de atendimento está sujeito e quais os requisitos a nível de proteção da informação? Quais softwares vêm sendo utilizados para fornecer esse tipo de serviço?

\section{Estratégia de pesquisa}

Para esta revisão sistemática foram utilizadas os repositórios digitais da CAPES, IEEE e Web of Science com o tema telemedicina e covid. Considerou-se apenas artigos escritos em português ou inglês, que tenham sido publicados entre 2018 e 2020 . O levantamento dos textos se deu por meio de Strings predefinidas que aglutinam os diversos conceitos relacionados à temática. A Tabela 1 apresenta as Strings de busca e a quantidade de trabalhos retornados em cada um dos repositórios. 
Tabela 1. Strings de Busca

\begin{tabular}{|c|c|c|}
\hline PERIÓDICO & STRING DE BUSCA & ARTIGOS \\
\hline CAPES & $\begin{array}{l}\text { video consulta AND covid } \\
\text { teleconsulta AND covid } \\
\text { telemedicina AND covid }\end{array}$ & 65 \\
\hline IEEE & 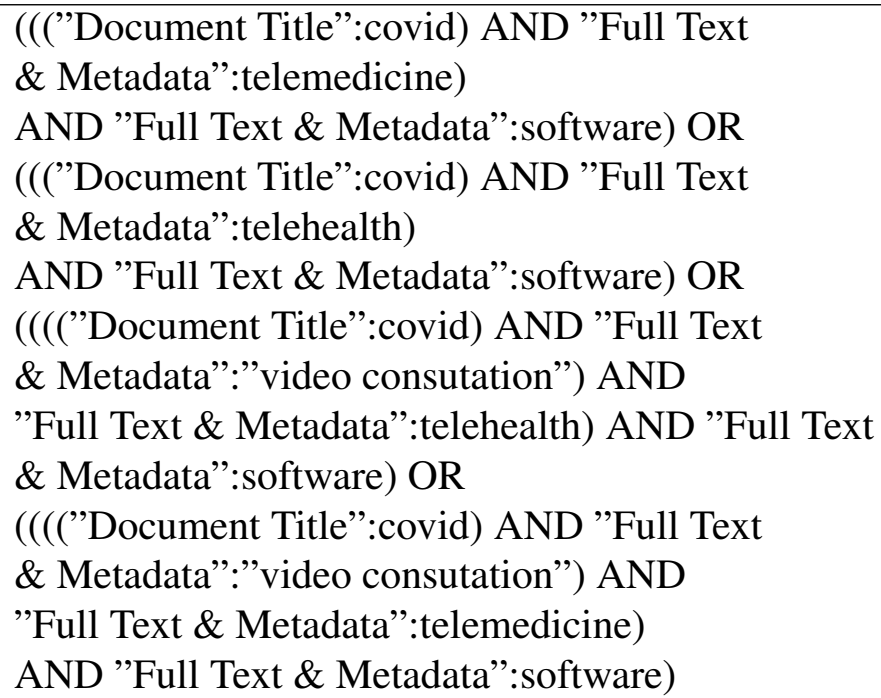 & 23 \\
\hline Web of Science & $\begin{array}{l}\text { (TS=(covid AND "video consultation"AND } \\
\text { telehealth AND software) ) OR } \\
\text { (TS=(covid AND telemedicine AND } \\
\text { software) ) } \\
\text { AND DOCUMENT TYPES: (Article) OR } \\
\text { (TS=(covid AND telehealth AND software) } \\
\text { AND DOCUMENT TYPES: (Article) OR } \\
\text { (TS=(covid AND "video consultation"AND } \\
\text { telemedicine AND software) ) }\end{array}$ & 20 \\
\hline
\end{tabular}

\section{Estratégia para Seleção dos Estudos}

Os 108 artigos retornados foram filtrados seguindo critérios de inclusão e exclusão predefinidos. Aplicados os critérios de exclusão, restaram um total de 51 artigos do conjunto inicial, os quais passaram por uma segunda filtragem por meio dos critérios de inclusão. Realizado tal procedimento, restaram os 10 trabalhos apresentados na Tabela 2, os quais serviram de roteiro para revisão sistemática.

- Critério de inclusão 1 (CI 1): Trabalhos que relatem experiências nacionais ou internacionais sobre o atendimento médico realizado por meio de videochamadas.

- Critério de inclusão 2 (CI 2): Trabalhos que abordem aspectos legais acerca da teleconsulta mediada por vídeo.

- Critério de inclusão 3 (CI 3): Artigos que relatem o desenvolvimento de novas ferramentas voltadas para atendimento médico remoto.

- Critério de Exclusão 1 (EX 1): Trabalhos que não foram escritos em português ou inglês.

- Critério de Exclusão 2 (EX 2): Trabalhos duplicados. 
- Critério de Exclusão 3 (EX 3): Trabalhos que descrevam soluções na área de telemedicina, cujo o foco seja diferente da teleconsulta.

Tabela 2. Artigos Selecionados

\begin{tabular}{|l|l|}
\hline \multicolumn{1}{|c|}{ TÍTULO } & \multicolumn{1}{|c|}{ CITAÇÃO } \\
\hline $\begin{array}{l}\text { Os desafios da telemedicina } \\
\text { frente a relação médico - paciente em tempos de Covid -19 }\end{array}$ & [Medeiros et al. 2020] \\
\hline $\begin{array}{l}\text { Telemedicina rural e COVID-19: ampliando o acesso } \\
\text { onde a distância já era regra }\end{array}$ & [Castro 2020] \\
\hline $\begin{array}{l}\text { Teleconsulta médica: os limites éticos e } \\
\text { o risco de negligência } \\
\text { informacional }\end{array}$ & [de Negreiros Calado et al. 2020] \\
\hline $\begin{array}{l}\text { Pilot Study Using Telemedicine Video Consultation } \\
\text { for Vascular Patients' Care During the COVID-19 Period }\end{array}$ & [Li et al. 2020] \\
\hline Online Patient Consultation & [Juhra et al. 2020] \\
\hline $\begin{array}{l}\text { Bridging the distance: Continuing psycho-oncological care } \\
\text { via video-consults during the COVID-19 pandemic }\end{array}$ & [Van der Lee 2020] \\
\hline $\begin{array}{l}\text { Telemedicine consultation for the clinical } \\
\text { cardiologists in the era of COVID-19: present and future. } \\
\text { Consensus document of the Spanish Society of Cardiology }\end{array}$ & [Barrios et al. 2020] \\
\hline $\begin{array}{l}\text { Use of telemedicine and virtual care for remote } \\
\text { treatment in response to COVID-19 pandemic }\end{array}$ & [Jnr 2020] \\
\hline $\begin{array}{l}\text { Development and Operation of a Video Teleconsultation } \\
\text { System Using Integrated Medical Equipment Gateway: } \\
\text { a National Project for Workers in Underserved Areas }\end{array}$ & [Park et al. 2020] \\
\hline $\begin{array}{l}\text { Applying software-defined } \\
\text { networking to support telemedicine health } \\
\text { consultation during and post Covid-19 era }\end{array}$ & [Jnr et al. 2020] \\
\hline
\end{tabular}

\section{Resultados da Revisão}

Os dez artigos selecionados serviram de base para responder às questões de pesquisa. Realizou-se a leitura e interpretação de todos eles de forma a sintetizar o conhecimento. Essa seção apresenta o resultado dessa análise. Dentre os trabalhos selecionados, quatro são relatos de experiência da aplicação de videochamadas na realização de consultas em diferentes países, incluindo o Brasil. Dois descrevem a implementação de ferramentas capazes de mediar ou melhorar esse tipo de atendimento. Três deles apresentam revisões de conceitos relacionados ao tema e recomendações para se realizar esse tipo de atendimento. Um descreve aspectos legislativos da teleconsulta no Brasil.

A Portaria $\mathrm{n}^{\circ} 467$, de 20 de março de 2020 foi utilizada de forma complementar para embasamento da resposta de segunda questão de pesquisa, relacionada às questões legais desse tipo de atendimento.

\subsection{QP1: Impactos e Limitações da Teleconsulta}

O uso de tecnologia da informação na mediação de atividades não é algo novo, mas sim uma prática já enraizada na sociedade. Entretanto, algumas atividades, por questões 
éticas, legais e culturais não eram praticadas em ambiente digital. É o caso do atendimento de pacientes de forma remota no Brasil e em alguns países do mundo.

A relação médico-paciente, quase sempre, se deu de forma presencial, não havendo antes da pandemia uma regulamentação clara de como transportar esse relacionamento para o ambiente digital. $\mathrm{O}$ atendimento por teleconsultas no Brasil, até março de 2020, era uma prática proibida e que sofria rechaço por parte das entidades médicas. A pandemia trouxe uma mudança abrupta de pensamento, que obrigou conselhos e profissionais de saúde a repensarem esse modelo tradicional. Com a publicação da Portaria n ${ }^{\circ}$ 467, de 20 de março de 2020, o atendimento remoto em atenção básica em saúde foi regulamentado de forma excepcional. Analisando experiências nacionais e internacionais que relatam a realização de consultas por videochamadas, observou-se que a tecnologia é apenas a base e que fatores humanos e econômicos de cada local influenciam bastante na eficiência dessa prática.

De início, observou-se que a grande maioria dos profissionais da área não possuíam o conhecimento técnico e a experiência para esse tipo de atendimento e tiveram que passar por treinamento para que pudessem exercê-la. Observou-se que muitos profissionais alegam que apesar da teleconsulta estar regulada nos seus países, não existem recomendações de como proceder uma teleconsulta, assim diferentes estratégias vem sendo adotadas. Para Juhra et al. (2020) essa falta de experiência com a tecnologia pode ser um dos grandes limitadores da sua difusão, visto que muitos profissionais já exercem diversas atividades e não teríam tempo de realizar novas capacitações.

Outro consenso entre profissionais é que apesar da teleconsulta permitir a comunicação remota médico-paciente, sofre de limitações no que se refere a realização de diversos tipos de exames clínicos e também físicos, não sendo assim um substituto integral do atendimento tradicional. Um dos estudos, cita a experiência da aplicação de teleconsultas com pacientes que realizavam atendimento psiquiátrico e conclui que a teleconsulta termina escondendo sintomas que seriam observados pelo terapeuta através de gestos e sinais corporais.

A falta de acesso à infraestrutura tecnológica também é um dos limitadores para difusão desse tipo de serviço, bem como a não familiariedade de pacientes com a tecnologia e aparelhos eletrônicos. Países onde a estrutura de redes e comunicação e o acesso da população à tecnologia é mais disseminado, possuem uma maior capacidade de implantar esse tipo de atendimento. Relatos de experiência da China, Alemanha, Coréia e Espanha se diferem de relatos brasileiros. No caso dos primeiros países, destaca-se que a presença de redes de alta velocidade e acesso da população à recursos tecnológicos são uma realidade factível. O uso de computadores, smatphones e softwares encontram-se bastante difundido e a população possui assim letramento digital satisfatório.

Por outro lado, no Brasil, como relatam Castro (2020) e Medeiros et al. (2020) o acesso às redes de alta velocidade encontra-se ainda limitado aos grandes centros e muitas pessoas, apesar de já terem tido contato com smartphones e computadores não possuem muita experiência com o uso de softwares utilizados para teleconsulta. Esses fatores podem limitar o acesso a esse tipo de atendimento em regiões rurais e remotas, locais que poderiam se beneficiar bastante com a nova prática e também o acesso daqueles cidadãos com baixo letramento digital. 
A teleconsulta realizada por videochamadas, apesar das limitações, também traz consigo uma série de benefícios relatados por médicos e pacientes. O principal deles seria a diminuição da circulação de pessoas em hospitais, algo de suma importância em tempos de isolamento social. Pacientes relatam que apesar do atendimento não estar sendo realizado de maneira presencial, sentem-se mais próximos do médico, alegando ter a sensação de suporte mais individualizado. Destaca-se também a comodidade ao se evitar deslocamentos e até mesmo viagens desnecessárias.

Levando em consideração todas as observações, destaca-se que teleconsultas realizadas por videochamadas podem ser bastante úteis para a realização de triagem e acompanhamento de pacientes, mas não um substituto integral do atendimento presencial. No Brasil, é preciso melhorar a infraestrutura tecnológica e criar políticas de acesso da população à esse tipo de atendimento, bem como ferramentas que facilitem o acesso de pessoas com menor letramento digital.

\subsection{QP2: Legislação e Recomendações}

Dos dez trabalhos analisados, cinco abordavam algum aspecto relativo à regulação de teleconsultas. Foi possível identificar que as recomendações quanto à proteção de dados e sigilo da informação são semelhantes, havendo algumas restrições no tipo de plataforma homologada para mediação da consulta e, no Brasil, a necessidade de assinaturas digitais para prescrição de receituários e atestados.

A confidencialidade é um dos requisitos de segurança mais importantes para a realização desse tipo de atendimento. Tanto os dados do paciente, quanto os do médico devem estar protegidos de acessos indevidos e as informações repassadas durante a consulta só devem ser entregues aos seus participantes. Isso significa que a criptografia ponta a ponta se torna um requisito para esse tipo de atendimento, sendo que grande parte das ferramentas de videochamadas da atualidade implementam essa característica.

No que tange a legislação, alguns países já possuíam leis que regulamentavam as consultas por vídeo antes da pandemia, caso da Alemanha e Coréia do Sul. Na Alemanha, desde o ano de 2017, permite-se prática de teleconsulta, havendo apenas a restrição de que o servidor utilizado para intermediar o atendimento esteja localizado dentro da união européia [Juhra et al. 2020]. Já a Coréia do Sul, desde 2018, trabalha com a informatização do atendimento por videochamadas, possuindo até mesmo um projeto governamental para acesso remoto de trabalhadores à saúde [Park et al. 2020]. Os Estados Unidos, assim como o Brasil, estabeleceram normativas em março de 2020, permitindo a utilização de softwares de videochamadas como Skype, Microsoft Teams, Face Time, entre outros enquanto durar a pandemia [Jnr 2020].

No Brasil a recomendação vigente foi definida pela Portaria $n^{\circ} 467$, de 20 de março de 2020 [da Saúde 2020], que trata do uso de tecnologia da informação na interação médico-paciente. $\mathrm{O}$ texto não menciona os tipos de plataformas que podem ser utilizadas para a mediação do atendimento, apenas destaca que o sigilo da informação e sua integridade sejam respeitadas. A portaria também define a necessidade do registro de dados de atendimento em um sistema de prontuário eletrônico, bem como o uso de certificados e chaves emitidos pela Infraestrutura de Chaves Públicas Brasileira (ICP-Brasil) para emissão de receitas médicas e atestados. Por fim, a normativa estabelece que o paciente deve ser informado da necessidade de exames físicos e das limitações do atendimento. 


\subsection{QP3: Softwares e Ferramentas}

Por ser uma prática adotada em caráter emergencial, não foram encontrados muitos relatos nacionais da aplicação de videochamadas na realização de consultas. Dos trabalhos analisados, apenas um descrevia esse tipo de experiência no Brasil [Castro 2020]. Outros dois relatavam experimentos na China [Li et al. 2020] e Coréia do Sul [Park et al. 2020]. Os estudos do Brasil e China utilizaram ferramentas de terceiros para hospedagem da teleconsulta, enquanto a solução coreana fazia uso de uma plataforma proprietária, desenvolvida para esse tipo de atendimento.

Observou-se nos trabalhos que utilizavam ferramentas de terceiros, uma prédisposição de escolha por plataformas bem difundidas e que funcionem em smartphones. Tal estratégia pode ser explicada pelo fato desse tipo de aparelho ter um custo mais baixo do que computadores e consequentemente um maior acesso por parte da população. Já a utilização de softwares conhecidos, como WhatsApp e WeChat, relatados nos dois estudos, se dá por questões de familiariedade entre o usuário e a plataforma, tornando mais simples a interação. Destaca-se que tais ferramentas não foram pensadas para esse fim, assim não fornecem opções para armazenamento de dados da consulta, do paciente ou do médico. Em ambos os artigos, descreve-se que após o atendimento se fazia necessária a transcrição das informações para prontuários físicos ou eletrônicos, bem como a emissão de receitas físicas, no caso do Brasil. Desse modo, soluções que agreguem essas funcionalidades poderiam agilizar o atendimento.

O trabalho coreano [Park et al. 2020] descreve uma alternativa completa para o atendimento médico à distância, contudo na forma de um consultório digital equipado com acessórios médicos interconectados à sensores. Segundo os autores, a solução não foi pensada para atendimentos em tempos de covid, visto que o paciente deve estar presencialmente em um ambiente equipado com com componentes médicos e tecnológicos específicos. Entretanto, traz contribuições no que tange a proteção de dados em teleconsulta, como o uso de redes virtuais privadas (VPN) e hospedagem de serviços de videochamadas em servidores locais.

Um ponto a ser destacado é que a qualidade do áudio e vídeo podem ser elencados como fatores determinantes para o sucesso ou fracasso de consultas remotas. Transmissões com baixa qualidade podem dificultar a troca de informações e até mesmo impedir o contato visual do profissional com o paciente, tornando a comunicação ineficiente.

\section{Considerações Finais}

A teleconsulta por meio de videochamadas é uma prática recente, capaz de fornecer atendimento médico especializado em tempos de isolamento social. Ela traz comodidade para médicos e pacientes, bem como segurança e praticidade, porém sofre de limitações. A falta de alternativas para realização de exames médicos, o conhecimento agregado necessário para sua implantação e a baixa disseminação de redes de alta velocidade são fatores que podem dificultar a disseminação desse tipo de atendimento no Brasil. Através da análise de diversos trabalhos sobre o tema, observou-se que as resoluções não estão tão claras e que relatos de experiência ainda são escassos. Todavia, foi possível identificar pontos que podem ser melhorados. Levando em consideração as normativas brasileiras, uma possível contribuição seria a integração de plataformas de teleconsulta em sistemas de informação com a capacidade de registro de prontuário eletrônico. Outra contribuição 
diz respeito ao desenvolvimento de alternativas para atendimento de pacientes com menor experiência com a tecnologia. Como trabalho futuro pretende-se definir uma arquitetura de teleconsulta integrada, bem como realizar a análise das principais plataformas de videochamadas.

\section{Referências}

Barrios, V., Cosín-Sales, J., Bravo, M., Escobar, C., Gámez, J. M., Huelmos, A., Cortés, C. O., Egocheaga, I., García-Pinilla, J. M., Jiménez-Candil, J., et al. (2020). Telemedicine consultation for the clinical cardiologists in the era of covid-19: present and future. consensus document of the spanish society of cardiology. Revista Española de Cardiología (English Edition), 73(11):910-918.

Castro, F. A. G. d. (2020). Telemedicina rural e covid-19: ampliando o acesso onde a distância já era regra. Rev. bras. med. fam. comunidade, pages 2484-2484.

da Saúde, M. (2020). Portaria no 467, de 20 de marÇo de 2020. [Online; acessado 26-Novembro-2020].

de Negreiros Calado, V., Lamy, M., et al. (2020). Teleconsulta médica: os limites éticos e o risco de negligência informacional. CADERNOS IBERO-AMERICANOS DE DIREITO SANITÁRIO, 9(3):89-122.

Dresch, A., Lacerda, D. P., and Júnior, J. A. V. A. (2015). Design science research: método de pesquisa para avanço da ciência e tecnologia. Bookman Editora.

Feitosa, H. (2019). A telemedicina em foco. Revista de Medicina da UFC, 59(1):6-8.

Jnr, B. A. (2020). Use of telemedicine and virtual care for remote treatment in response to covid-19 pandemic. Journal of Medical Systems, 44(7):1-9.

Jnr, B. A., Nweke, L. O., and Al-Sharafi, M. A. (2020). Applying software-defined networking to support telemedicine health consultation during and post covid-19 era. Health and technology, pages 1-9.

Juhra, C., Ansorg, J., Back, D. A., John, D., Kuckuck-Winkelmann, A., Raschke, M., Osterhoff, G., Pförringer, D., and Scherer, J. (2020). Online patient consultation. Z Orthop Unfall, 158(4):345-350.

Li, H.-L., Chan, Y. C., Huang, J.-X., and Cheng, S. W. (2020). Pilot study using telemedicine video consultation for vascular patients' care during the covid-19 period. Annals of vascular surgery, 68:76-82.

Medeiros, A. M. d. B., Beltrão, B. C. R., and Neto, O. (2020). Os desafios da telemedicina frente a relação médico - paciente em tempos de covid -19. Research, Society and Development, 9(10).

Park, H. S., il Kim, K., Soh, J. Y., Hyun, Y. H., Lee, B. E., Lee, J. H., Jo, J. G., Lee, H. C., and Kim, H. S. (2020). Development and operation of a video teleconsultation system using integrated medical equipment gateway: a national project for workers in underserved areas. Journal of Medical Systems, 44(11):1-14.

Van der Lee, Marije L. e Schellekens, M. P. J. (2020). Bridging the distance: Continuing psycho-oncological care via video-consults during the covid-19 pandemic. PsychoOncology, 29(9):1421-1423. 